\title{
Stability of the Albanese Fibration on the Cartwright-Steger Surface
}

\author{
Vincent Koziarz and Sai-Kee Yeung*
}

\begin{abstract}
We verify that the Albanese fibration of the Cartwright-Steger surface is stable, answering a problem left open in [4].
\end{abstract}

The purpose of this note is to resolve a question left open in [4] about the semi-stability of the Albanese fibration of the Cartwright-Steger surface, denoted by $X$. The CartwrightSteger surface is a smooth complex two ball quotient of Euler number 3 and first Betti number $b_{1}=2$. It is known that 3 is the smallest possible number achievable as the Euler number of a smooth surface of general type. The Cartwright-Steger surface is the only such surface with a non-trivial first Betti number, as explained in Theorem 4.2 of [1] and the erratum there. The other smooth surfaces of general type with Euler number 3 are the fake projective planes, classified in [5,8, which have vanishing first Betti number, and there are 100 of those. We refer the readers to [2,4 6] for basic geometric properties of $X$.

Since $h^{1,0}(X)=1$, there is a non-trivial Albanese map. Let $\alpha: X \rightarrow E$ be the Albanese fibration, where $E$ is an elliptic curve and is the Albanese variety of $X$. It is proved in [4] that the fibration is reduced and the genus of a generic fiber is 19. A natural question left open in [4] is whether the Albanese fibration is semi-stable, cf. Remark 5.6 of [4]. Once the fibration is proved to be semi-stable, it follows from the fact that we are considering a complex two ball quotient with a fibration as above that the fibration is stable.

The problem turns out to be subtle, and defied conventional algebraic geometric methods after repeated attempts. We have to combine the group theoretical results of [4] and explicit equations of [2] to achieve our purpose.

Theorem 1. The Albanese mapping $\alpha: X \rightarrow E$ is a stable fibration in the sense of Deligne-Mumford.

Recall the following facts about $X$ from [4]. The automorphism group is given by $\operatorname{Aut}(X)=\mathbb{Z}_{3}$ and the fixed point set of a generator of $\operatorname{Aut}(X)$ consists of 9 isolated

Received September 23, 2020; Accepted November 29, 2020.

Communicated by Jungkai Alfred Chen.

2020 Mathematics Subject Classification. 14J29, 14Q10, 14J50.

Key words and phrases. Albanese fibration, special surfaces, stability.

Yeung was partially supported by a grant from the National Science Foundation.

*Corresponding author. 
points, three of type $\frac{1}{3}(1,1)$ denoted by $O_{i}, i=1,2,3$, all lying in one fiber of $\alpha$, and six of type $\frac{1}{3}(1,2)$, denoted by $Q_{j}, j=1, \ldots, 6$, distributed evenly among two different fibers of $\alpha$. The following statement is found in Remark 5.6 of [4], with the details given in Proposition 5 of 3$]$.

Lemma 2. [4] Assume that $\alpha$ is not stable. Then there is only one singular fiber, and there is exactly one tacnode singularity on the singular fiber. The singularity is then one of the points $Q_{j}, j=1, \ldots, 6$ listed above.

In [2], explicit equations are found to describe $X$ as a surface in $P_{\mathbb{C}}^{9}$ with coefficients in the rational number field $\mathbb{Q}$, consisting of 85 polynomial equations of degree 2 and 3 . As a result, the complex conjugation of $X$ with respect to the natural complex structure gives a complex surface biholomorphic to itself.

Lemma 3. 22 Denote by $\tau$ the restriction of the complex conjugation of $P_{\mathbb{C}}^{9}$ to $X$. Then $\tau: X \rightarrow X$ is a diffeomorphism of $X$ and the fixed point set is a real subvariety of $X$.

Let $F$ be the fixed point set of $\tau$ on $X$.

Lemma 4. The set $F$ contains $O_{i}, i=1,2,3$, but does not contain $Q_{j}, j=1, \ldots, 6$.

Proof. We present two different arguments. The first one find explicit coordinates of the fixed points with help from Matlab, and the second one more directly from the computations related to [2].

We explain first the first method. The action of $\mathbb{Z}_{3}$ on $X$ can be described as follows. According to Remark 5.3 of [2], the generator $g_{3}$ acts by

$$
\begin{aligned}
& g_{3}\left[U_{0}: U_{1}: U_{2}: U_{3}: U_{4}: U_{5}: U_{6}: U_{7}: U_{8}: U_{9}\right] \\
= & {\left[U_{0}: U_{1}: U_{2}: U_{3}: a U_{4}: a U_{5}: a U_{6}: b U_{7}: b U_{8}: b U_{9}\right], }
\end{aligned}
$$

where $a=\zeta_{3}$ is a primitive cube root of unity, and $b=a^{2}$.

It follows that the fixed points of $g_{3}$ have one of the following types,

(1) $\left[U_{0}: U_{1}: U_{2}: U_{3}: 0: 0: 0: 0: 0: 0\right]$,

(2) $\left[0: 0: 0: 0: U_{4}: U_{5}: U_{6}: 0: 0: 0\right]$,

(3) $\left[0: 0: 0: 0: 0: 0: 0: U_{7}: U_{8}: U_{9}\right]$

From Remark 5.3 of [2], we already know that the points $O_{i}, i=1,2,3$ are given by the three points $[0: 0: 0: 0: 0: 0: 0: 1: 0: 0],[0: 0: 0: 0: 0: 0: 0: 0: 1: 0]$, $[0: 0: 0: 0: 0: 0: 0: 0: 0: 1]$, corresponding to points of type (3). In terms of the 85 explicit equations defining $X$ given in [2], the manifold $X$ is the set of complex solutions of the equations $q_{k}(U)=0, k=1, \ldots, 85$ for $U \in P_{\mathbb{C}}^{9}$. The subvariety $F$ is the set of 
real solutions of the 85 equations. Trying to find solutions of the types (1) to (3), we use Matlab and find that apart of $O_{i}, i=1,2,3$ as above, there are six other points of type (1), which correspond to the points $Q_{j}, j=1, \ldots, 6$. There is no fixed point of type (2). The coordinates $U=\left[U_{0}: U_{1}: U_{2}: U_{3}: U_{4}: U_{5}: U_{6}: U_{7}: U_{8}: U_{9}\right]$ of $Q_{j}, j=1, \ldots, 6$ are given in terms of three conjugate pairs of algebraic numbers, with $U_{2} / U_{0}$ given by

$$
-0.0927 \pm 0.1987 \sqrt{-1}, \quad \pm 32.0785 \sqrt{-1}, \quad 0.0927 \pm 0.1987 \sqrt{-1}
$$

in decimals respectively. Hence we may assume that

$$
Q_{2}=\overline{Q_{1}}, \quad Q_{4}=\overline{Q_{3}}, \quad Q_{6}=\overline{Q_{5}}
$$

after renaming if necessary. The coordinates were found with the help of Ling Xu using Matlab. Since the coordinates are not real, the points do not lie on $F$.

Alternatively, Cartwright explained to us that we may argue without using Matlab but with symbolic computation as follows. It follows from setting $U_{0}=1$ and $U_{i}=0$ for $i=4, \ldots, 9$ as in (1) above in the set of 85 explicit equations given in [1,2] that $U_{3}^{2}$ satisfies the equation $z^{3}+\left(15597 z^{2}\right) / 32+(641385 z) / 4096+884547 / 4096=0$, from which one easily sees that $U_{3} / U_{0}$ cannot be real.

Recall that the Albanese map $\alpha: X \rightarrow E$ is defined by $\alpha(\xi)=\int_{x_{o}}^{\xi} \omega(\bmod \Lambda)$, where $\omega$ is the holomorphic one form on $X, x_{0} \in X$ is a fixed point on $X$, the integration is taken over any path on $X$ and $\Lambda$ is the lattice generated by $\int_{\gamma} \omega$ as $\gamma$ varies over loops of $X$ generated by $\pi_{1}(X)$. The lattice $\Lambda$ takes care of the ambiguity in the choice of path of integration in the definition above. Choose $x_{o}$ to be a fixed point on $X$ with real coordinates, e.g., $O_{j}$ for some $j=1,2,3$.

Recall also that the Albanese variety $\alpha(X)=E$ is given by $\mathbb{C} /\left(\mathbb{Z}+\zeta_{3} \mathbb{Z}\right)$, where $\zeta_{3}$ is a primitive cube root of unity. This follows from the discussions in [4]. From the classification of finite automorphism groups of elliptic curves, $E$ has a Weierstrass form given by $z_{2}^{2}=z_{1}^{3}+1$, cf. [7, p. 34] and [10, Theorem III.10.1], which is the unique elliptic curve with automorphism group containing $\mathbb{Z}_{3}$. In this case, $\operatorname{Aut}(E)=\mathbb{Z}_{6}=\left\langle\zeta_{6}\right\rangle$, which acts on $E$ by $\zeta_{6} \cdot\left(z_{1}, z_{2}\right)=\left(\zeta_{6}^{2} z_{1}, \zeta_{6}^{3} z_{2}\right)$.

In the following, we realize $X$ as a subvariety of $P_{\mathbb{C}}^{9}$ as given in [2], and $E$ with the Weierstrass representation in $P_{\mathbb{C}}^{2}$ as above. Then both $X$ and $E$ are invariant by the complex conjugation on the respective projective spaces and the Albanese mapping $\alpha$ will be discussed in terms of the realizations. We let $U=\left[U_{j}\right]$ and $Z=\left[Z_{\beta}\right]$ be the homogeneous coordinates on the ambient manifolds $P_{\mathbb{C}}^{9}$ and $P_{\mathbb{C}}^{2}$ respectively. $P_{\mathbb{C}}^{9}$ is covered by coordinate charts $\mathcal{U}_{i}=\left\{U \mid U_{i} \neq 0\right\}$ with inhomogeneous coordinates $\xi_{i, j}=U_{j} / U_{i}$ for $0 \leq j \neq i \leq 9$. Let $\mathcal{U}_{i, j k}$ be the set of points $p \in X$ so that the Jacobian matrix of the defining equations of $X$ with respect to $\left(\xi_{i, j}, \xi_{i, k}\right)$ at $p$ has complex rank 2 . Then the set 
of $\left(\mathcal{U}_{i, j k}, \xi_{i, j}, \xi_{i, k}\right)$ for $0 \leq i \leq 9,0 \leq j<k \leq 9, j \neq i, k \neq i$, forms a coordinate system on $X$. For simplicity of notation, we would suppress $i$ and simply denote $\xi_{i, j}$ by $\xi_{j}$. The same convention is used for $E$. Let $\mathcal{V}_{\gamma}=\left\{Z \mid Z_{\gamma} \neq 0\right\}$ and $z_{\gamma, \beta}=Z_{\beta} / Z_{\gamma}$ for $0 \leq \beta \neq \gamma \leq 2$. The set of $\left(\mathcal{V}_{\gamma, \beta}, z_{\gamma, \beta}\right)$ for $0 \leq \gamma, \beta \leq 2, \beta \neq \gamma$ forms a coordinate system on $E$. Again, we suppress $\gamma$ for notational simplicity. In affine coordinates of $E$, we may use $z=z_{1}$ or $z_{2}$ as coordinate functions in the Weierstrass representation of $E$. Writing $\xi_{j}=u_{j}+i v_{j}$ and $z_{\beta}=x_{\beta}+i y_{\beta}$, the Albanese map $\alpha$ is given by $\alpha(\xi)=z(\xi)=x(u, v)+i y(u, v)$, regarding $x, y$ as functions of $u$ and $v$. Although the construction of these explicit coordinates is elementary, we explain it in detail since we have to use them in the proof below.

Lemma 5. In terms of the realization above, the Albanese map a satisfies the following.

(a) $\bar{\alpha}(\bar{\xi})=\sigma(\alpha(\xi))$ for some $\sigma \in \mathbb{Z}_{6}$ in the automorphism group of $E$ fixing the origin.

(b) Let $p \in E$. Then the complex conjugate of a fiber of $\alpha$ at $p$ is the fiber at $\sigma^{-1}(\bar{p})$ for some $\sigma \in \mathbb{Z}_{6}$.

Proof. We describe the Albanese map in terms of the coordinates above. From the fact that $\alpha$ is holomorphic, we know that $x_{u_{j}}=y_{v_{j}}, x_{v_{j}}=-y_{u_{j}}$ from Cauchy-Riemann equations, where $j=1,2$. It follows that $x_{u_{j}}=(-y)_{\left(-v_{j}\right)}, x_{\left(-v_{j}\right)}=-(-y)_{u_{j}}$. This implies that $\bar{\alpha}(\bar{\xi})$ is holomorphic in $\xi$ and hence is a non-trivial holomorphic map of $X$ to its Albanese torus $E$. From the functorial properties of the Albanese map, such a holomorphic map is unique up to a biholomorphism of $E$. Hence once the based point $x_{o}=O_{i}$ is fixed, $\alpha$ is unique up to $\sigma \in \mathbb{Z}_{6}$. Hence $\bar{\alpha}(\bar{\xi})=\sigma \circ \alpha(\xi)$.

(a) leads to $\sigma \circ \alpha(\bar{\xi})=\bar{\alpha}(\xi)$ or $\bar{\xi} \in \alpha^{-1}\left(\sigma^{-1}(\bar{\alpha}(\xi))\right)$. Hence by considering the set of $\xi$ with $\alpha(\xi)=p$, the complex conjugate $\overline{\alpha^{-1}(p)}$ of the fiber at a point $p \in E$ is actually the fiber of $\alpha$ at $\sigma^{-1}(\bar{p}) \in E$. Hence (b) follows.

Proof of Theorem 1. Assume on the contrary that the Albanese fibration is not semistable, so that according to Lemma 2, there is a tacnode singularity at $Q_{j}$ on the fiber $L=\alpha^{-1}(p)$ (with $p$ one of the fixed points of the $\mathbb{Z}_{3}$ action on $E$ ). From Lemma 5(b), $\tau(L)$ is a fiber of $\alpha$ and hence, $\tau\left(Q_{j}\right)$, which is different from $Q_{j}$ by Lemma 4 , is another singularity. Note that the fibration map $\pi$ has singularity at a point $Q$ if and only if the same happens at $\tau(Q)$, where $\tau$ is a diffeomorphism. This contradicts Lemma 2 .

We remark that at the finishing touch of the paper, it is drawn to our attention that there is another proposed independent proof of the stability of the Albanese fibration by Carlos Rito [9, which also relies on the work of [2,4], but in a different manner. 


\section{Acknowledgments}

The authors would like to thank Donald Cartwright and Lev Borisov for helpful discussions, and to thank Ling Xu for her help with Matlab. They would also like to thank the referees for helpful suggestions. The second author thanks the hospitality of Université de Bordeaux, at which the research here was partly conducted.

\section{References}

[1] L. Borisov, http://www.math.rutgers.edu/ borisov/CS/.

[2] L. A. Borisov and S.-K. Yeung, Explicit equations of the Cartwrightx-Steger surface, Épijournal Géom. Algébrique 4 (2020), Art. 10, 13 pp.-12.

[3] D. I. Cartwright, V. Koziarz and S.-K. Yeung, On the Cartwright-Steger surface, long version, arXiv:1412.4137.

[4] _ On the Cartwright-Steger surface, J. Algebraic Geom. 26 (2017), 655-689.

[5] D. I. Cartwright and T. Steger, Enumeration of the 50 fake projective planes, C. R. Math. Acad. Sci. Paris 348 (2010), no. 1-2, 11-13.

[6] _ Finding generators and relations for groups acting on the hyperbolic ball, preprint.

[7] D. Husemöller, Elliptic Curves, Second edition, Graduate Texts in Mathematics 111, Springer-Verlag, New York, 2004.

[8] G. Prasad and S.-K.Yeung, Fake projective planes, Invent. Math. 168 (2007), no. 2, 321-370; Addendum, Invent. Math. 182 (2010), no. 1, 213-227.

[9] C. Rito, Surfaces with canonical map of maximum degree, arXiv:1903.03017.

[10] J. H. Silverman, The Arithmetic of Elliptic Curves, Second edition, Graduate Texts in Mathematics 106, Springer, Dordrecht, 2009.

[11] S.-K. Yeung, Foliations associated to harmonic maps on some complex two ball quotients, Sci. China Math. 60 (2017), no. 6, 1137-1148; Erratum, Sci. China Math. 63 (2020), no. 8, 1645.

Vincent Koziarz

Université de Bordeaux, IMB, CNRS, UMR 5251, F-33400 Talence, France

E-mail address: vincent.koziarz@math.u-bordeaux.fr 
Sai-Kee Yeung

Purdue University, West Lafayette, IN 47907, USA

E-mail address: yeung@math.purdue.edu 